\title{
Influencia del ámbito, rural o urbano, en la percepción de los apoyos y las necesidades de las personas cuidadoras en el hospital
}

\section{Influence of environment, rural or urban, in the perception of the supports and needs of caregivers in hospitals}

\author{
B. Gorlat-Sánchez ${ }^{1}$, M.P. García-Caro ${ }^{2}$, P. Peinado-Gorlat ${ }^{3}$, M. Expósito-Ruizi ${ }^{1}$, \\ A. Quero-Rufián ${ }^{4}$, F. Cruz-Quintana ${ }^{5}$
}

\section{RESUMEN}

Fundamento. Tras implementar un Plan Funcional con medidas de apoyo a personas cuidadoras en dos hospitales de Granada, se analizan la eficacia y el efecto del medio en la percepción y las necesidades de la persona cuidadora.

Material y método. Se realizó un estudio cualitativo, fenomenológico, a través de entrevistas semi-estructuradas. Participaron 45 personas cuidadoras incluidas en el Plan Funcional. Se midieron además apoyo social percibido, función familiar y nivel de ansiedad mediante los cuestionarios Duke-unc, Apgar familiar y Escala de Goldberg, respectivamente. El análisis de los datos cualitativos se llevó a cabo mediante codificación abierta, axial y selectiva, y los cuestionarios mediante estadística descriptiva.

Resultado. Las personas cuidadoras del ámbito urbano valoraron más los apoyos que les facilitaban la vida en el hospital y los apoyos sociales para el domicilio y las del ámbito rural los apoyos hospitalarios orientados a educación en cuidados para cuando estuviesen en su domicilio, todos ellos vinculados a la personalización de cuidados. Las alteraciones personales por el cuidado están presentes en ambos ámbitos aunque difieren en las repercusiones psíquicas y en el tipo de fármacos y su consumo. Existen diferencias en cuanto a los apoyos necesarios y las dificultades encontradas.

Conclusiones. El ámbito donde habitualmente viven las personas cuidadoras condiciona sus percepciones del cuidado y algunas de sus necesidades en el hospital. Se deberán revisar las medidas del Plan Funcional cuestionadas, redefinir algunas prioridades e incluir medidas adicionales en función de la situación familiar de las personas cuidadoras. Palabras clave. Dependencia. Hospitalización. Cuidadores. Zona rural. Zona urbana.

\begin{abstract}
Background. After the Functional Plan was implemented with support measures for caregivers in hospitals of Granada (Spain), the effectiveness and impact of environment on the perception and needs of caregivers was analysed.

Materials and methods. A qualitative, phenomenological study was made through semi-structured interviews. A total of 45 caregivers included in the Functional Plan participated. In addition, perceived social support, family functioning, and the anxiety level was measured, respectively, through the Duke-UNC, Apgar Family, and Goldberg Scale questionnaires. The data were qualitatively analysed by open, axial, and selective coding, and the questionnaires by descriptive statistics.

Results. The caregivers of the urban setting valued more the support that made their life easier in the hospital and the social support at home, while caregivers in rural settings valued the hospital support oriented towards education in care for life after returning home, all linked to the personalization of care. The personal disturbances due to care occurred in all the settings, although differences appeared in psychic repercussions and in the type of drugs and their consumption. There were differences in terms of support needed and the difficulties encountered.

Conclusions. The setting where caregivers live determine their perceptions of the care and some of their needs in the hospital. The measures questioned in the Functional Plan should be reviewed and some priorities should be redefined, including additional measures, depending on the family situation of the caregivers.
\end{abstract}

Key words. Dependency. Hospitalization. Caregivers. Rural. Urban.
1. Hospital Virgen de las Nieves. Granada.

2. Facultad de Ciencias de la Salud. Universidad de Granada. Granada.

3. Fundación Pública Andaluza para la Investigación Biosanitaria de Andalucía Oriental. Granada.

4. Escuela Universitaria de Enfermería Virgen de las Nieves. Granada.

5. Facultad de Psicología. Universidad de Granada. Granada.

Recepción: 20 de junio de 2013

Aceptación provisional: 29 de julio de 2013

Aceptación definitiva: 9 de septiembre de 2013

\author{
Correspondencia: \\ Berta Gorlat-Sánchez \\ Hospital Santa Ana \\ Avenida Enrique Martín Cuevas, 0 S/N \\ 18600 Motril \\ Granada. España \\ bertagorlat7@hotmail.com
}

Esta investigación fue financiada en convocatoria pública por la Consejería de Salud y Bienestar de la Junta de Andalucía en la modalidad de Proyectos de investigación, con el código de expediente PI: 0406/09 durante 30 meses, no existiendo conflictos de intereses. 


\section{INTRODUCCIÓN}

Las tendencias actuales tanto en España como en el resto de Europa apuntan a un incremento en los próximos años de la proporción de personas mayores dependientes ${ }^{1}$.

En España, la Encuesta sobre Discapacidad, Autonomía personal y situaciones de Dependencia (EDAD 2008) ${ }^{2}$, sitúa a Andalucía entre las comunidades con tasas más elevadas de dependencia. Con un número estimado de 424.921personas dependientes $^{3}$, la atención de estas personas constituye un reto no solo para la política sanitaria y social sino también para las familias que han de asumir el cuidado . $^{2}$

Como respuesta a este reto, en concreto respecto de la atención a los cuidadores, la Consejería de Salud desarrolló en el año 2005 el Plan de Mejora de la atención a las personas cuidadoras (en adelante PC), basado en el Decreto de Apoyo a las Familias Andaluzas $^{5}$, y en la Ley de Promoción de la Autonomía Personal y Atención a las Personas en Situación de Dependencia y a las Familias $^{6}$, plan que supuso un avance, especialmente en atención primaria, aunque no tanto en los hospitales en los que hay que reconocer que, no se desarrollaron las medidas suficientes para dar cobertura a las necesidades de las PC como beneficiarias del sistema sanitario en base a la Ley de Dependencia ${ }^{7-8}$.

El perfil de PC más frecuente en Andalucía, según el informe "Discapacidad y dependencia en Andalucía" publicado en 2011, es el de una mujer, con una edad media superior a 50 años, casada, con estudios primarios o inferiores, que suele ser la hija o la cónyuge de la persona dependiente. Se calcula en más de 87.000 las hijas que atienden a alguno de sus padres o a ambos. Entre los cónyuges destaca también la mujer que cuida a su esposo; siendo cada vez más frecuente el varón cuidador de elevada edad ${ }^{9}$.

Según la literatura científica revisada sobre el tema, las necesidades de las PC están condicionadas por factores propios como el género, la edad y el nivel de formación, pero también por otros como el peso de las tradiciones de cada lugar, las circunstancias culturales y los apoyos sociales y sanitarios recibidos, además de las repercusiones negativas en la salud como consecuencia del cuidado ${ }^{10-13}$. En estos otros factores juega un importante papel el ámbito en el que habitualmente viven. Minguela y Camacho identifican en su estudio "Cuidados formales e informales en Andalucía", las diferencias entre el ámbito rural y el urbano que pueden influir en la asunción del cuidado ${ }^{14}$. Según datos mostrados por el IMSERSO en 2011, el 25\% de las personas mayores dependientes viven en municipios rurales, aunque en cifras absolutas los dependientes son sobre todo urbanos, considerando núcleos urbanos aquellos con población mayor a 10.000 habitantes ${ }^{10}$.

En este contexto, el estudio: "La población rural en España: de los desequilibrios a la sostenibilidad social", señala que en el ámbito rural se dan una serie de circunstancias como el éxodo rural producido hace unas décadas, el descenso de la fecundidad y una esperanza de vida mayor a la del ámbito urbano, que hacen que la población esté más envejecida y que exista un alto porcentaje de dependencia severa, lo que puede suponer peores condiciones para las PC. También se identifica que, a menudo, en el ámbito rural los apoyos familiares son menores, los servicios públicos no se adecuan a los caracteres propios de este medio y siguen muy arraigados estereotipos culturales tradicionales entre los que se considera que la actividad del cuidado, al igual que el trabajo doméstico, ha de ser asumido por la mujer, aunque con cierta variabilidad, dependiendo del tamaño de la población, de su aislamiento y de los servicios a los que tiene acceso $^{15}$.

Con objeto de completar el desarrollo del Plan de Mejora de la atención a las PC y hacerlo extensivo a la hospitalización, se puso en marcha un plan funcional pionero, con medidas específicas de apoyo, en dos hospitales de Granada, el hospital General Virgen de las Nieves y el hospital de Baza. Ambos hospitales son públicos, si bien, el primero es regional, ubicado en la ciudad 
de Granada, y el segundo comarcal, encontrándose en un ámbito rural.

El Plan Funcional implementado incluye medidas de apoyo dirigidas a las $\mathrm{PC}$ con el objetivo de facilitarles su labor, formarlas, protegerlas y atenderlas adecuadamente durante el periodo de hospitalización de la persona a la que cuidan habitualmente. Para la elaboración del Plan, se tomaron como punto de partida los resultados de un estudio previo, realizado en 2005 con cuidadoras informales del hospital General Virgen de las Nieves, en el que se hacían visibles las actividades de las PC en el hospital, sus necesidades y demandas ${ }^{8}$.

En el Plan Funcional se consideró PC a "aquella persona sobre la que recae la responsabilidad del cuidado cuando el paciente esté en el domicilio y de quien depende la persona dependiente para poder satisfacer sus necesidades básicas"16-17. Se implementaron las siguientes medidas: acogida, información y personalización de cuidados a través de la asignación de enfermera y auxiliar referentes; identificación con tarjetas personalizadas, educación en cuidados individualizada y grupal en talleres formativos, accesos prioritarios, apoyo al descanso y aseo, coordinación de citas y pruebas complementarias, atención sanitaria, farmacéutica y social a la PC y apoyo hostelero.

En este plan funcional participan además de las enfermeras y las auxiliares de enfermería, otros colectivos del hospital, como administrativos, trabajadores sociales, fisioterapeutas, técnicos especialistas, médicos, farmacéuticos y celadores.

Tras un tiempo de implementación de dos años en el hospital General Virgen de las Nieves y de uno en el hospital de Baza, se realizó una investigación con el objetivo de evaluar la eficacia de las medidas de apoyo llevadas a cabo. Para ello se diseñó una estrategia encaminada a conocer la experiencia directa de las PC incluidas en el programa, que se complementó con el análisis de la situación personal y familiar de las mismas.

Concretamente, este trabajo se centra en el análisis del efecto del ámbito en la percepción de las medidas de apoyo en el hospital y si estas cubren las necesidades de la PC. Por la influencia directa que pueden ejercer, también se han identificado las repercusiones personales del cuidado, el consumo de fármacos, las ayudas con las que cuentan y las dificultades para el cuidado percibidas por ellas.

\section{MATERIAL Y MÉTODOS}

Se realizó un estudio cualitativo, enfocado a analizar en profundidad las experiencias de las $\mathrm{PC}$, partiendo del supuesto ontológico de que la realidad se construye socialmente a través del lenguaje, siendo este esencial para captar significados ${ }^{18}$. El método de investigación seguido se basa en la Teoría Fundamentada. Se utilizaron como estrategias el muestreo teórico y el método comparativo constante.

El período de estudio fue desde enero de 2010 a junio de 2012 .

Los sujetos de estudio fueron PC identificadas como tales en las plantas de hospitalización de adultos de los servicios de Medicina Interna, Oncología, Digestivo, Cirugía y Neumología de ambos hospitales.

Los criterios de inclusión fueron: haber tenido experiencias de hospitalización como PC, anteriores a la implementación del Plan Funcional, en el mismo hospital; aceptar participar en el estudio; y estar en ese momento identificada por su enfermera referente como PC. Para ello habían de cumplir los criterios referidos al paciente y a la PC que figuran en el Plan Funcional representados en la tabla 1.

La muestra fue intencional. La selección de los participantes se basó en los perfiles obtenidos según la combinación de aquellos criterios de heterogeneidad que, en la búsqueda de evidencias, mostraron mayor efecto en la variabilidad discursiva. Se consideraron como criterios de heterogeneidad: el sexo, la edad, el parentesco con la persona cuidada, el ámbito donde viven habitualmente, la situación laboral, el nivel de estudios y las cargas familiares.

Se buscó la saturación en todos los perfiles en los que fue posible. 
Tabla 1. Criterios de inclusión como PC en el hospital. Plan Funcional de medidas de apoyo a las PC en los hospitales Virgen de las Nieves y Baza (Granada)

Criterios referidos al paciente: (al menos 2 de los 3 criterios siguientes):

1. Ingreso previsto con estancias superiores a 10 días en áreas médicas y 5 días en áreas quirúrgicas.

2. Índice de Barthel $<40$, mantenido durante un periodo de al menos 7 días.

3. Índice de Pfeiffer $\geq 5$ errores.

Criterios referidos a la PC: (al menos 2 de los 3 criterios siguientes):

1. Asumir el rol de PC en el domicilio.

2. Presentar alto nivel de sobrecarga (índice de esfuerzo del cuidador $\geq 7$ puntos).

3. Capacidad para recibir información en cuidados y realizarlos adecuadamente después en el domicilio.

Quedan incluidas directamente como PC en el hospital todas aquellas personas identificadas como cuidadoras de gran discapacitado (CGD).

La identificación como PC la realiza cada enfermera referente tras la valoración inicial en las primeras 24 horas de ingreso del paciente.

Para la recogida de datos de las variables se elaboró un documento "ad hoc". Se recogieron variables que permitieron identificar características personales, familiares y sociales de cada una de las PC entrevistadas.

Las variables apoyo social percibido, función familiar y ansiedad, se midieron a través de los siguientes cuestionarios: Duke-unc, para valorar el apoyo social percibido; Apgar familiar, para la percepción de la función familiar y Escala de Goldberg, para el cribado de ansiedad/depresión que consta de 2 subescalas, una para la ansiedad y otra para la depresión. En este estudio solo se utilizó la subescala de ansiedad para identificar la existencia de ansiedad en la PC, y la información obtenida se refirió a las dos últimas semanas, no puntuando los síntomas de duración inferior a dos semanas o de intensidad leve. Todos estos cuestionarios han sido validados en población española ${ }^{19-21}$.

En el registro del consumo de fármacos se recogió la medicación que la $\mathrm{PC}$ consumía regularmente.

Para las entrevistas semiestructuradas, se elaboró un guión con las siguientes temáticas: tiempo cuidando, repercusiones físicas y psíquicas del cuidado, dificultades sociales y sanitarias percibidas en su vida cotidiana, cambios y apoyos percibidos en el hospital con respecto a ingresos anteriores, nivel de satisfacción con los apoyos recibidos y propuestas de cambios y mejoras del Plan Funcional.

Tanto los datos referidos a las distintas variables y los cuestionarios se recogieron en una sola sesión. Las entrevistas tuvieron una duración que osciló entre 20 y 60 minutos.

Los informantes clave a través de las cuales se identificaron las posibles candidatas al estudio fueron las supervisoras de las unidades de hospitalización incluidas en la investigación. En cada caso, tras comprobar que cumplía los criterios de inclusión en la investigación y que su perfil era adecuado para ser incluida en el estudio, la informante clave realizó la presentación entre la PC y la investigadora. A continuación se les explicaban los objetivos del estudio, el procedimiento que se seguiría, en qué consistiría su colaboración, cuánto tiempo aproximadamente les podía suponer y cuál iba a ser la utilización de los datos. Además se le hacía entrega de la hoja informativa y se procedía a la firma del consentimiento informado. La hora de realización de la entrevista fue pactada en cada caso y el lugar 
de realización fue siempre un espacio situado en el propio hospital.

Para el análisis de las entrevistas se siguió la secuencia propuesta por Corbin y Strauss. El proceso de análisis de la información se inició mediante el denominado "microanálisis" o "análisis detallado línea por línea", realizando una codificación abierta; después se definieron las propiedades de cada categoría y se relacionaron entre ellas por medio de codificación axial, finalmente se realizaron comparaciones a través de la codificación selectiva con el fin de generar teoría ${ }^{22}$.

Los códigos de las entrevistas se agruparon en torno a 6 categorías: satisfacción con los apoyos en el hospital, quejas y propuestas de mejora a las medidas del plan funcional, alteraciones personales por el cuidado, ayudas y dificultades encontradas para el cuidado.

Se realizó un análisis diferencial entre informantes pertenecientes a los distintos ámbitos (rural y urbano), que permitió destacar las diferencias fundamentales en sus discursos. Se estructuró de acuerdo a las categorías establecidas filtrando las distintas variables mediante el programa informático Atlas ti. 5.0.

Se realizó análisis descriptivo de las variables con expresión de los porcentajes de éstas.

\section{RESULTADOS}

Se identificaron 1.119 PC (899 en el hospital Virgen de las Nieves y 220 en el hospital de Baza). Cumplían criterios de inclusión en el estudio 223 personas, de ellas 29 rehusaron directamente participar en el estudio, en 43 casos no fue posible realizar la entrevista y en 51 casos se produjo la muerte del paciente antes de que fuese posible la entrevista. De los 100 posibles participantes se entrevistó a 45 , representativos de la mayoría de los perfiles que se pretendía estudiar (de algunos perfiles posibles no se encontraron casos). De ellos 18 casos se recogieron en el hospital de Baza y 27 en el hospital Virgen de las Nieves de Granada. Los perfiles de las PC entrevistadas se muestran en la tabla 2.

Tabla 2. Perfiles de las PC participantes.

\begin{tabular}{|c|c|c|c|c|c|c|c|c|c|}
\hline \multicolumn{2}{|c|}{ Perfiles PC entrevistadas } & \multicolumn{4}{|c|}{ Ámbito rural } & \multicolumn{4}{|c|}{ Ámbito urbano } \\
\hline & Sexo & Mujeres & Mujeres & Hombres & Hombres & Mujeres & Mujeres & Hombres & Hombres \\
\hline & Nivel de estudios & $\mathrm{Se} / \mathrm{EP}$ & ES/ESP & $\mathrm{Se} / \mathrm{EP}$ & ES/ESP & $\mathrm{Se} / \mathrm{EP}$ & ES/ESP & $\mathrm{Se} / \mathrm{EP}$ & ES/ESP \\
\hline \multirow{2}{*}{$\begin{array}{c}\leq \mathbf{5 5} \text { años } \\
(44,4 \%)\end{array}$} & Con carga familiar & 1 & 4 & - & 3 & 1 & 2 & - & 1 \\
\hline & Sin carga familiar & 1 & 2 & 1 & 1 & 2 & - & - & 1 \\
\hline \multirow{3}{*}{$\begin{array}{c}>\mathbf{5 5} \text { años } \\
(55,6 \%)\end{array}$} & Con carga familiar & 3 & - & 1 & - & 4 & - & - & 1 \\
\hline & Sin carga familiar & 3 & - & 4 & 1 & 5 & - & 2 & 1 \\
\hline & Totales & 8 & 6 & 6 & 5 & 12 & 2 & 2 & 4 \\
\hline
\end{tabular}

Se (sin estudios). EP (estudios primarios). ES (estudios secundarios). ESP (estudios superiores).

Del total de PC entrevistadas el 60\% pertenecían al ámbito rural y el $40 \%$ al urbano. Las distintas variables que los describen y las diferencias de porcentajes entre los dos ámbitos se recogen en la tabla 3 . 
Tabla 3. Variables incluidas en el estudio diferenciadas en porcentajes según ámbito.

\begin{tabular}{|c|c|c|}
\hline \multirow{2}{*}{ Variables } & \multicolumn{2}{|c|}{ Ambito } \\
\hline & Rural (60\%) & Urbano (40\%) \\
\hline $\begin{array}{l}\text { Sexo } \\
\text { Hombres/ Mujeres }\end{array}$ & $\begin{array}{l}40,7 \% \text { hombres } \\
59,3 \% \text { mujeres }\end{array}$ & $\begin{array}{l}33,3 \% \text { hombres } \\
66,7 \% \text { mujeres } \\
\end{array}$ \\
\hline $\begin{array}{l}\text { Edad } \\
\quad \text { Menores de } 55 \text { años/ Mayores de } 55 \text { años }\end{array}$ & $\begin{array}{l}55,6 \%>55 \text { años } \\
44,4 \%<55 \text { años }\end{array}$ & $\begin{array}{l}66,7 \%>55 \text { años } \\
33,3 \%<55 \text { años }\end{array}$ \\
\hline $\begin{array}{l}\text { Nivel de estudios } \\
\quad \text { Sin estudios/Primarios } \\
\text { Secundarios/ Superiores }\end{array}$ & $\begin{array}{l}22,2 \% \text { sin estudios } \\
37,0 \% \text { estudios primarios } \\
25,9 \% \text { estudios secundarios } \\
14,8 \% \text { estudios superiores } \\
\end{array}$ & $\begin{array}{l}11,1 \% \text { sin estudios } \\
55,6 \% \text { estudios primarios } \\
22,2 \% \text { estudios secundarios } \\
11,1 \% \text { estudios superiores } \\
\end{array}$ \\
\hline $\begin{array}{l}\text { Situación laboral } \\
\text { Activo / Inactivo } \\
\text { Activos: trabajadores/as por cuenta ajena, } \\
\text { trabajadores por cuenta propia y amas de } \\
\text { casa. } \\
\text { Inactivos: jubilados, parados, estudiante. }\end{array}$ & $\begin{array}{l}70,4 \% \text { activos } \\
29,6 \% \text { inactivos }\end{array}$ & $\begin{array}{l}44,4 \% \text { activos } \\
55,6 \% \text { inactivos }\end{array}$ \\
\hline $\begin{array}{l}\text { Cargas familiares } \\
\text { Sí/ No } \\
\text { Si: Hijos/as o convivientes en el hogar. }\end{array}$ & $\begin{array}{l}\text { 48,1\% sí } \\
51,9 \% \text { no }\end{array}$ & $\begin{array}{l}50 \% \text { sí } \\
50 \% \text { no }\end{array}$ \\
\hline $\begin{array}{l}\text { Parentesco } \\
\text { cónyuges } \\
\text { hijos/as } \\
\text { hermanos/as } \\
\text { madre/padre } \\
\text { otros (especificar) } \\
\end{array}$ & $\begin{array}{l}55,6 \% \text { cónyuges } \\
29,6 \% \text { hijos/as } \\
7,4 \% \text { hermanos/as } \\
3,7 \% \text { madre/padre } \\
3,7 \% \text { sobrinos/as }\end{array}$ & $\begin{array}{l}50,0 \% \text { cónyuges } \\
38,9 \% \text { hijos/as } \\
5,6 \% \text { hermanos/as } \\
5,6 \% \text { madre/padre }\end{array}$ \\
\hline $\begin{array}{l}\text { CGD } \\
\text { Personas identificadas con la Tarjeta }+ \\
\text { cuidado (Cuidadora de gran discapacitado) }\end{array}$ & $50 \%$ & $50 \%$ \\
\hline $\begin{array}{l}\text { Apoyo social percibido } \\
\text { Cuestionario Duke- unk } \\
\geq 32 \text { apoyo social percibido normal. } \\
\text { < } 32 \text { apoyo social percibido bajo. }\end{array}$ & $\begin{array}{l}44,4 \% \text { normal } \\
55,6 \% \text { bajo }\end{array}$ & $\begin{array}{l}61,1 \% \text { normal } \\
38,9 \% \text { bajo }\end{array}$ \\
\hline $\begin{array}{l}\text { Función familiar } \\
\text { Cuestionario Apgar familiar } \\
\text { Normofuncional: } 7-10 \text { puntos } \\
\text { Disfuncional leve: } 3-6 \\
\text { Disfuncional grave: } 0-2 \text {. }\end{array}$ & $\begin{array}{l}59,3 \% \text { normal } \\
33,3 \% \text { disfunción leve } \\
7,4 \% \text { disfunción grave }\end{array}$ & $\begin{array}{l}61,1 \% \text { normal } \\
38,9 \% \text { disfunción leve } \\
0 \% \text { disfunción grave }\end{array}$ \\
\hline $\begin{array}{l}\text { Ansiedad } \\
\text { Escala de Goldberg-Subescala de Ansiedad } \\
\text { Ansiedad } \geq 4 \\
\text { No ansiedad }<4\end{array}$ & $\begin{array}{l}51,9 \% \text { ansiedad } \\
48,1 \% \text { no ansiedad }\end{array}$ & $\begin{array}{l}44,4 \% \text { ansiedad } \\
55,6 \% \text { no ansiedad }\end{array}$ \\
\hline $\begin{array}{l}\text { Consumo de fármacos } \\
\text { Analgésicos/ Antidepresivos/ } \\
\text { Antihipertensivos/ Anticoagulantes/ } \\
\text { Ansiolíticos/Tranquilizantes/ Antiarritmicos/ } \\
\text { Antidiabéticos/ Antidepresivos/ Laxantes/ } \\
\text { Antiinflamatorios. }\end{array}$ & $\begin{array}{l}\text { 18,5\% no } \\
\text { 81,5\% sí: más significativos: } \\
\text { - Analgésicos: } 57,9 \% \\
\text { - Antidepresivos: } 40 \% \\
\text { - Antihipertensivos: } 50 \% \\
\text { - Ansiolíticos/ } \\
\quad \text { tranquilizantes: } 58,8 \% \\
\end{array}$ & $\begin{array}{l}\text { 16,7\% no } \\
\text { 83,3\% sí: más significativos: } \\
\text { - Analgésicos: } 42,1 \% \\
\text { - Antidepresivos: } 60 \% \\
\text { - Antihipertensivos: } 50 \% \\
\text { - Ansiolíticos/ } \\
\text { tranquilizantes: } 41,2 \% \\
\end{array}$ \\
\hline
\end{tabular}

\section{Satisfacción con los apoyos recibidos en el hospital}

Con respecto a esta categoría, se encontraron diferencias en relación al descanso, la educación en cuidados, el apoyo social, la gestión de citas-traslados, el aseo y el apoyo en hostelería.

Las PC del ámbito urbano expresaron con mayor frecuencia satisfacción con los apoyos en descanso, aseo, dieta alimenticia y apoyo social. 
- Necesitaba salir de aquí y descansar en mi cama, entonces ellas me dijeron que me fuera tranquila y se quedaron ellas vigilándolo, antes no era así. E_l $A U$

- Además hay un aseo y unas duchas que están muy bien porque yo no me ducho en la de los enfermos... te dan la toalla aquí en la planta y es como un hotel. E_26 AU

- Cuando no me traían la comida... aparte del gasto económico, tenía que perder esa media hora. La ventaja ahora... que te lo ponen al lado y no tienes que moverte de ahí... esto, contribuye a facilitarte un poco tu labor. $E \_2 A U$

En el ámbito rural las PC expresaron satisfacción fundamentalmente con las actividades de educación en cuidados y gestión de citas-traslados. Mientras que con frecuencia rechazaron los apoyos en aseo y dieta.

- La practicanta del pueblo los fines de semana no viene... tendríamos que ir a otro pueblo... yo lo que quiero es que mi enfermera me enseñe en el hospital para luego curarlo yo en la casa. E_15 AR

- Hasta mi auxiliar me ha querido dar toallas y jabón por si me quiero duchar aquí, pero para eso yo prefiero ir a mi casa. E_28 AR

- Incluso me han dicho de darme la comida, aunque yo les he dicho que lo agradezco pero que prefiero utilizar ese tiempo para salir y despejarme. E_43 AR

En general la satisfacción con los apoyos en el hospital fue alta en las PC de ambos ámbitos, destacando especialmente la satisfacción con la asignación de enfermera y auxiliar referentes.

- Ahora cuando ingresas tienes tu enfermera que te hace un montón de preguntas del enfermo y también pregunta por la cuidadora, y también tienes tu auxiliar que está pendiente de ti... te dan una tarjeta especial para reconocerte... esto no estaba antes cuando hemos venido al hospital. E_6AU

- Desde que ingresamos esta vez, vino J.C., me dijo que él era nuestro enfermero y MA nuestra auxiliar, me ex- plicaron que ahora a las cuidadoras como yo les están ayudando mientras están en el hospital, me dio esta tarjeta... desde entonces me están enseñando a cuidar mejor de mi marido, me dan la comida y me han mandado a la enfermera de casos y a la trabajadora social que han estado hablando conmigo... yo estoy muy agradecida porque me están ayudando mucho y diciendo cosas que yo no sabía. E_27AR

- He estado muchas veces en el hospital con mis padres, e incluso con mi hermano y esta vez he notado que hay cosas nuevas... Cuando ingresas tu enfermera te hace un montón de preguntas del enfermo pero también pregunta por la cuidadora... se han interesado mucho por los dos, por él y por mí, eso nunca lo habían hecho así, y me ha gustado. E_30AR

\section{Quejas y propuestas de mejora a las medidas de apoyo del Plan Funcional}

En esta categoría, las diferencias encontradas se centraron en el apoyo al descanso, al aseo y a la intimidad.

Algunas PC del ámbito urbano expresaron quejas sobre la incomodidad de los sillones, especialmente aquellas con estancias prolongadas en el hospital.

- El sillón es malísimo, es muy duro. Llevo durmiendo 39 noches en él con cuatro almohadas que me dieron las enfermeras. $E_{-} 15 \mathrm{AU}$

- Los sillones deberían ser más cómodos o poner una pequeña cama plegable sobre todo si ya llevas tantos días como yo. E_14 AU

También emitieron algunas quejas acerca de la ubicación de los baños y las duchas, y propusieron que se ubicaran más cerca.

- No voy al baño ni a las duchas nuestras porque están lejos de la habitación. E_16 AU

- Si le digo la verdad incluiría que en cada planta pudiese haber un sitio donde ir al baño y que no tuviésemos que bajar. E_16 AU 
Entre las PC del ámbito rural fue más frecuente la demanda del derecho a la intimidad, proponiendo en diversos casos que las habitaciones fuesen individuales.

- ... su compañero pesa 130 kilos, y cada vez que se mueve se le va toda la ropa, y yo que siempre he sido una persona que mira mucho por eso... me da mucho reparo. E_12 AR

- Si es posible que nos dejen en habitaciones individuales. $E_{-} 7 \mathrm{AR}$

\section{Alteraciones personales por el cuidado}

Se identificaron diferencias en cuanto a repercusiones psíquicas, físicas, alteraciones familiares y laborales. Las categorías y las citas se muestran comparando los ámbitos en la tabla 4 .

Las "repercusiones psíquicas" más frecuentemente en el ámbito rural fueron: ansiedad, miedo y sentimiento de soledad. El porcentaje de ansiolíticos/tranquilizantes consumidos por las PC del ámbito rural fue del $58,8 \%$ frente al 41,2\% del urbano. Los resultados obtenidos de la subescala de ansiedad mostraron más presencia de ansiedad en las PC del ámbito rural (51,9\% y 44,4\% respectivamente).

En el ámbito urbano se identificaron con mayor frecuencia: depresión, irritabilidad y sentimiento de culpa por institucionalizar a la persona dependiente. Coincidió con un porcentaje de consumo de antidepresivos mayor (60\% frente a $40 \%$ en el medio rural).

En cuanto a las "repercusiones físicas" se encontraron diferencias con respecto a dolores óseos y musculares, haciendo referencia a ellos con mayor frecuencia las $\mathrm{PC}$ del ámbito rural. Se registró un consumo de analgésicos en el 57,9\% de los casos del medio rural.

En los discursos de las PC se identificaron con frecuencia expresiones sobre "repercusiones en las relaciones familiares" a consecuencia del cuidado que alteraban la vida doméstica, aunque no se encontraron diferencias entre los ámbitos. En este aspecto, los resultados del cuestionario Apgar familiar mostraron que entre el 33,3\% (rural) y el $38,9 \%$ (urbano) de casos presentaban niveles de disfunción leve, mien- tras que los niveles de disfunción grave se produjeron solo en el ámbito rural en un $7,4 \%$ de casos (Tabla 3).

Con respecto a "repercusiones laborales", en los casos estudiados del ámbito rural se produjeron 2 reducciones de jornada, 2 modificaciones en los horarios del trabajo, un caso de baja laboral como consecuencia de repercusiones psicológicas por la asunción del cuidado y un caso con intención de abandonar el trabajo para dedicarse al cuidado.

En el ámbito urbano solo en UN caso se produjo abandono del trabajo para dedicarse al cuidado y en otro caso se pidió excedencia temporal.

\section{Ayudas y dificultades}

Se encontraron diferencias en cuanto a las ayudas familiares entre los dos ámbitos. En el medio rural, con frecuencia, las personas mayores asumían el cuidado en solitario, aún estando también enfermas.

Se encontraron más apoyos familiares en el ámbito urbano que en el rural (Tabla 4).

En bastantes casos las PC entrevistadas tenían ayudas sociales y sanitarias como consecuencia de la aplicación de la Ley de Dependencia, siendo más frecuentes en el ámbito urbano que en el rural.

Los resultados del cuestionario Dukeunc mostraron un apoyo social percibido más bajo en las PC rurales que en las urbanas (55,6\% y 38,9\%) (Tabla 3 ).

Se identificaron dificultades sociales y sanitarias diferentes. En el ámbito rural las dificultades fueron por falta de información y asesoramiento y resaltaron la falta de cobertura sanitaria por la dispersión geográfica y la distancia al centro sanitario.

En el ámbito urbano la principal dificultad social identificada fue la demora en recibir ayudas cuando se encontraban en el domicilio (ayudas económicas para dedicar al cuidado, centros de día, respiros familiares...) y como dificultades sanitarias expresaron falta de tutela en la realización de las actividades básicas de la vida diaria cuando se encontraban en el domicilio, y un perfil poco adecuado en algunas personas de asistencia en domicilio (Tabla 4). 
Tabla 4. Códigos y citas según el ámbito de las categorías: Alteraciones personales por el cuidado, ayudas y dificultades.

\begin{tabular}{|c|c|c|}
\hline Categoría & Ámbito rural & Ámbito urbano \\
\hline & $\begin{array}{l}\text { Sintomas psíquicos: ansiedad, miedo y } \\
\text { sentimiento de soledad } \\
\text { Consumo de ansiolíticos/tranquilizantes } \\
58,8 \% \text { frente a } 41,2 \% \text { en el medio urbano }\end{array}$ & $\begin{array}{c}\text { Síntomas psíquicos: depresión, irritabilidad } \\
\text { y sentimiento de culpa } \\
\text { Consumo de antidepresivos } 60 \% \\
\text { frente a } 40 \% \text { en el medio rural }\end{array}$ \\
\hline & $\begin{array}{l}\text { - Me afecta más de lo que yo pensaba. Me } \\
\text { causa mucha ansiedad estar todo el día de- } \\
\text { dicado a él... me da miedo de que le pase } \\
\text { algo, he tenido que dejar mis cosas... es una } \\
\text { obligación a la que no estoy acostumbrado } \\
\text { y me viene muy grande. E_41 AR } \\
\text { - Se ve uno muy solo en esto. (Pausa larga y } \\
\text { llanto). E_21 AR } \\
\text { - Llevo cuidando a mi marido } 5 \text { años... tiene } \\
\text { cáncer de colon, lo operaron y le han hecho } \\
\text { una ostomía... él no lo acepta (llanto)... y } \\
\text { yo tampoco... me da miedo, pero él no se } \\
\text { puede enterar porque si no se hunde, y aquí } \\
\text { estoy sufriendo esto en soledad. E_ } 9 \text { AR }\end{array}$ & $\begin{array}{l}\text { - A mí me da mucha pena (llanto). Por la noche lo oigo } \\
\text { llorar... y yo también lloro...no hablamos... ¿y qué nos } \\
\text { vamos a decir?, tampoco dormimos... con la inquietud } \\
\text { que uno tiene cómo va a dormir... yo tengo depresión, } \\
\text { pero no lo quiero demostrar y estoy aquí haciéndome la } \\
\text { fuerte. E_16 AU } \\
\text { _ Psicológicamente esto me está afectando mucho, estoy } \\
\text { siempre irritada, por un lado no puedo dejarlo y por } \\
\text { otro también me tengo que cuidar yo, por mi y por mi } \\
\text { familia... tengo a mis hijos y mi marido que también me } \\
\text { necesitan... y yo es que estoy jubilada por mí proble- } \\
\text { ma... y a pesar de eso tengo que cuidarlo, no me queda } \\
\text { otro remedio. E_25 AU } \\
\text { - Fisicamente estoy cansado, pero sobre todo esto afecta } \\
\text { psíquicamente...lo he pasado fatal mientras la tenía en } \\
\text { la residencia... ella decía que estaba contenta, pero a mí } \\
\text { me remordía la conciencia y me sentía culpable, así que } \\
\text { la tuve que sacar. E_19 AU }\end{array}$ \\
\hline
\end{tabular}

Síntomas físicos: dolores óseos y musculares

Consumo de analgésicos: $57,9 \%$ en el medio rural frente a $42,1 \%$ en el medio urbano.

\section{Alteraciones personales por el cuidado}

- El otro día se me cayó y no podía levantarla, empecé a arrastrarla y me crujió la cintura, desde entonces me cuesta mucho moverla, además como he trabajado tanto en el campo, tengo la espalda fatal. E_21 AR
- Me siento mejor... aunque tengo dolores. Porque yo tengo fibromialgia y también tengo un par de hernias discales, así que de dolores sí que estoy muy mal... pero con calmantes voy tirando. $E_{-} 11 \mathrm{AU}$

Repercusiones familiares (no se identifican diferencias entre ámbitos)

- Como esto es tan largo hasta nuestra relación se ha resentido. Hasta ahora de vez en cuando venía mi cuñada, pero ya no porque ella dice que está mala y no puede. Yo ya sé que esto es para mí y ni me planteo buscar a alguien. $E_{-} 7 \mathrm{AR}$

- Ella es muy vieja, y muy cabezona, con unas costumbres muy antiguas, mi hermano también va a su aire y esto ya no es una familia, ya no puedo más. E_29 AR (Disfunción grave)

- Nos concedieron una plaza en el centro de día..., necesitábamos una ayuda porque estábamos todos de los nervios, hasta hemos discutido entre nosotros cuando nunca lo habíamos hecho y desde entonces la relación no es la misma. $E \_26 \mathrm{AU}$

- Uno se cree que puede llevarlo todo pero... hay muchos momentos en los que tienes bajones, y al final esto afecta a toda la familia mucho. E_6 $6 U$

\section{Repercusiones laborales (no se identifican diferencias entre ámbitos)}

- Desde hace seis meses me he pedido una reducción de jornada para su cuidado y solo trabajo de diez a una y media. E_33 AR

- Nunca me he dado de baja laboral y espero no tener que hacerlo, pero si llega el momento tengo claro que mi hijo es antes que mi trabajo, el director del instituto lo sabe y mis compañeros también, y lo comprenden... E_38 AR

- Si esto empeora no voy a poder seguir trabajando... a ver cómo nos las vamos a arreglar. E_ 31 AR

- Tienes que tomar la decisión, y dejas todo, he dejado el trabajo... él te necesita a ti, y prácticamente desde que te levantas hasta que te acuestas, vives pendiente a esa persona, y tu vida se enfoca todo a través de él. E_2 AU

- Cuánto ha cambiado mi vida, mucho... pedí una excedencia en el trabajo... ya no existe el tiempo de ocio.. no me libro del estrés puesto que no se desconecta, aunque quieras no puedes desconectar. E_6 AU 


\begin{tabular}{|c|c|c|}
\hline Categoría & Ámbito rural & Ámbito urbano \\
\hline \multirow{4}{*}{ Ayudas } & $\begin{array}{l}\text { Ayudas familiares: con frecuencia } \\
\text { personas mayores asumían el cuidado en } \\
\text { solitario, aún estando también enfermas. }\end{array}$ & Ayudas familiares: más apoyos familiares. \\
\hline & $\begin{array}{l}\text { - Nos vamos apañando, nuestros hijos nos } \\
\text { llaman por teléfono y nos preguntan... Algu- } \\
\text { nas veces estoy que no puedo ni hacer la } \\
\text { comida, cada vez estoy mal delgada... será } \\
\text { por el azúcar... . pero lo tengo que cuidar, a } \\
\text { veces le pongo las pastillas porque las co- } \\
\text { nozco por lo gordas que son cada una y por } \\
\text { el color, pero me da miedo equivocarme... } \\
\text { tengo cada caja en un lugar distinto, cada } \\
\text { una en un cajón, y así las distingo. E_23 AR }\end{array}$ & $\begin{array}{l}\text { - Tengo dos nietos maravillosos, que me ayudan con el } \\
\text { cuidado del abuelo. De vez en cuando se quedan una } \\
\text { semana con él y yo me voy a descansar a la Manga del } \\
\text { mar menor... y eso me tiene fuerte, fuerte. E_13 AU }\end{array}$ \\
\hline & $\begin{array}{c}\text { Ayudas sociales y sanitarias por la Ley } \\
\text { de Dependencia } \\
\text { Duke-unc: apoyo social normal: } 44,4 \% / / \\
\text { apoyo social bajo } 55,6 \%\end{array}$ & $\begin{array}{c}\text { Ayudas sociales y sanitarias por la Ley de } \\
\text { Dependencia: mayores que en el ámbito rural. } \\
\text { Duke-unc: apoyo social normal: } 61,1 \% \text { // apoyo social } \\
\text { bajo: } 38,9 \%\end{array}$ \\
\hline & $\begin{array}{l}\text { - Mira que hemos dado vueltas... me mandó } \\
\text { el médico del centro de salu luego vinieron } \\
\text { a la casa con la enfermera y una muchacha } \\
\text { que nos hizo muchas preguntas y estuvo } \\
\text { viendo como está el baño, la casa y todo, } \\
\text { pero quedaron en darnos una respuesta y } \\
\text { todavía no han dicho nada y ya hace bas- } \\
\text { tante. E_24 AR }\end{array}$ & $\begin{array}{l}\text { - Tengo una muchacha que viene } 2 \text { días en semana por } \\
\text { la mañana y me ayuda a bañarlo. La trabajadora social } \\
\text { del ayuntamiento me había ofrecido un centro de día } \\
\text { para que se vaya por la mañana y venga a las } 5 \text { de la } \\
\text { tarde. E_l6 AU } \\
\text { - A la casa viene una mujer por la mañana que nos man- } \\
\text { da el ayuntamiento, todo esto nos lo arregló la enfer- } \\
\text { mera del centro de salud que nos conoce desde hace } \\
\text { muchos años, ello me hizo esta tarjeta y me dijo que } \\
\text { la enseñe siempre que vaya a algún sitio de la sani- } \\
\text { dad, que con ella me atienden antes... y es verdad así } \\
\text { lo hago. E_ } 10 \mathrm{AU}\end{array}$ \\
\hline \multirow{4}{*}{ Dificultades } & $\begin{array}{c}\text { Dificultades sociales } \\
\text { Falta de información y asesoramiento }\end{array}$ & $\begin{array}{c}\text { Dificultades sociales } \\
\text { Demora en recibir ayudasDificultades sociales }\end{array}$ \\
\hline & $\begin{array}{l}\text { - He encontrado muchas dificultades, no sa- } \\
\text { bía dónde tenía que ir... tampoco nadie te } \\
\text { dice lo que se puede hacer. } E_{-} 19 \text { AR } \\
\end{array}$ & $\begin{array}{l}\text { - Me dijeron que me ponían en lista de espera para la } \\
\text { residencia y que tardaría unos dos años... cómo voy a } \\
\text { esperar tanto tiempo... } E_{-} 25 \mathrm{AU} \text {. }\end{array}$ \\
\hline & $\begin{array}{l}\text { Dificultades sanitarias } \\
\text { Falta de cobertura sanitaria por dispersión } \\
\text { geográfica y distancia al centro sanitario }\end{array}$ & $\begin{array}{c}\text { Dificultades sanitarias } \\
\text { Falta de tutela en la realización de las ABVD cuando se } \\
\text { encontraban en el domicilio y perfil poco adecuado de } \\
\text { algunas personas de asistencia en domicilio }\end{array}$ \\
\hline & $\begin{array}{l}\text { - Allí no hay médico ni enfermero, solo vie- } \\
\text { nen } 2 \text { veces a la semana... hace } 20 \text { días co- } \\
\text { gió un resfriado y tuvo fiebre... y cuando por } \\
\text { fin vino el médico lo envió rápidamente al } \\
\text { hospital. E_23 AR } \\
\text { - Estamos muy lejos del centro más próximo, } \\
\text { y me da miedo no saber qué hacer si pasa } \\
\text { algo urgente. E_15 AR }\end{array}$ & $\begin{array}{l}\text { - Yo lo hago como Dios me da a entender, aquí no viene } \\
\text { nadie a ver si lo haces bien o no. E_13 AU } \\
\text { - Las chicas que vienen a casa no saben manejar a estos } \\
\text { enfermos... no me fio de ellas... eso es algo que habrá } \\
\text { que mejorar. E_15 AU. }\end{array}$ \\
\hline
\end{tabular}

\section{Condicionantes de la evaluación de la eficacia de las medidas de apoyo a la PC en el hospital}

Del análisis de las interrelaciones entre categorías y entre códigos se deduce una hipótesis explicativa sobre las relaciones entre los elementos que condicionan la evaluación de la eficacia de las medidas de las PC (Fig. 1). Se encontraron dos factores que intervienen directamente en la eficacia de las medidas de apoyo a las PC en el hospital 
en relación a la respuesta a sus necesidades. Por una parte, y como elemento externo al hospital, se identificó que las necesidades de las PC cambiaban en función de sus alteraciones personales por el cuidado. En este sentido se comprobó la influencia directa del ámbito en el que vivían habitualmente, ya que en función de éste cambiaba el sentido de las ayudas y las dificultades encontradas para asumir el cuidado.
Por otra parte, las PC expresaron que las medidas de apoyo en el hospital habían cubierto sus necesidades, siendo su nivel de satisfacción alto a pesar de existir algunas quejas y realizar propuestas de mejora. Se identificó como factor interno del hospital y elemento mejor valorado, la personalización de cuidados a través de la asignación de enfermera y auxiliar referentes a cada PC.

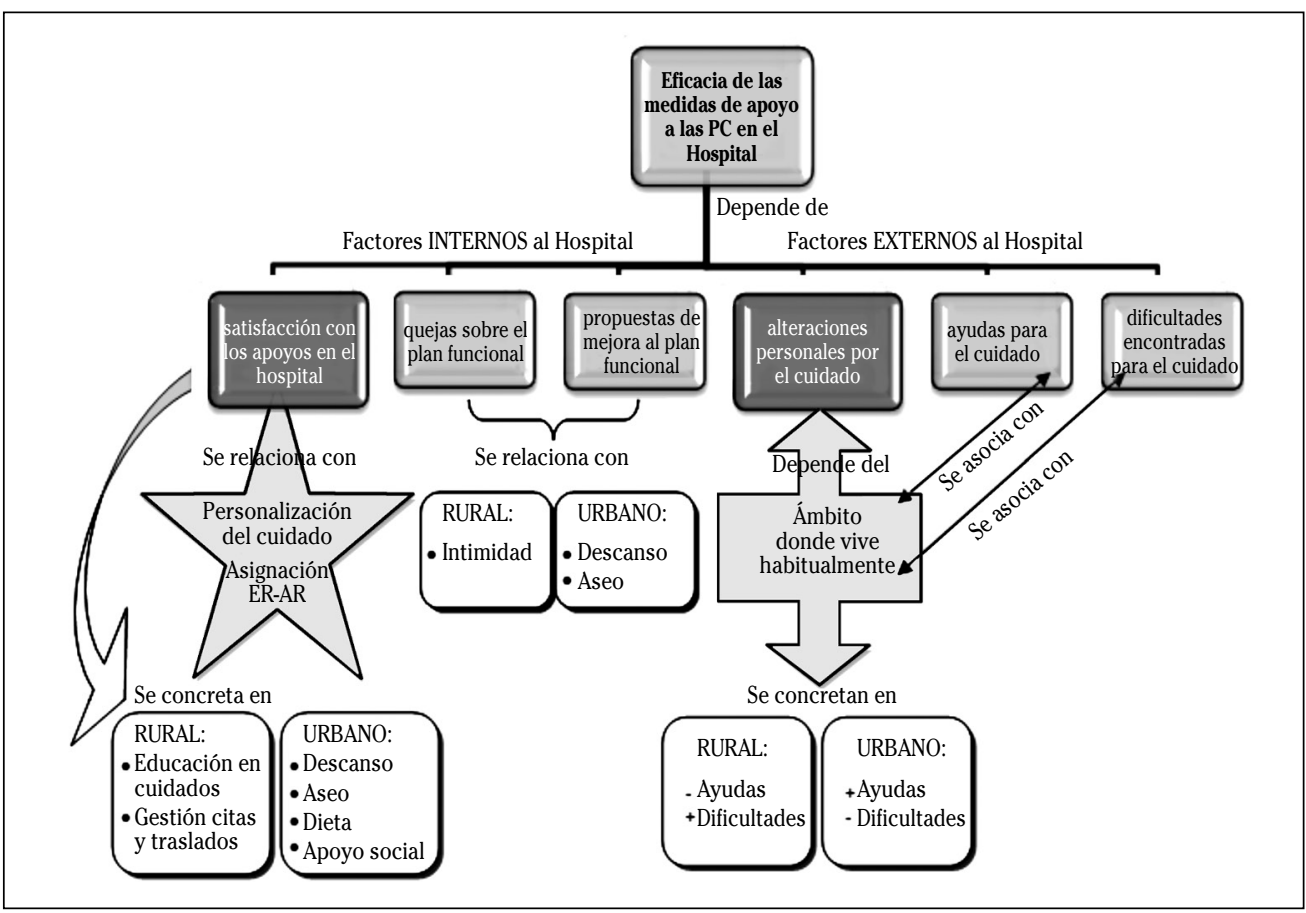

Figura 1: Factores influyentes en la eficacia de las medidas de apoyo a las PC en el hospital, categorías centrales y elementos fundamentales de éstas.

\section{DISCUSIÓN}

En general, las PC entrevistadas expresaron un alto nivel de satisfacción con las medidas del Plan Funcional. En su discurso se identificó que las PC del ámbito urbano eran más exigentes en cuanto a aquellos elementos que les facilitaban la vida en el hospital, por lo que valoraban especialmente los apoyos hospitalarios en descanso, aseo y alimentación y los apoyos sociales para cuando estuviesen en su domicilio; sus quejas y propuestas de mejora sobre las medidas de apoyo del Plan Funcional siguieron esa misma línea. En cambio, las PC del ámbito rural, valoraban especialmente los apoyos recibidos en el hospital que servirían para facilitarles después la vida cuando se encontraran en su domicilio, ya que identificaban tener menores apoyos sociales que en el ámbito urbano, lo que les generaban mayores niveles de ansiedad, miedo y sentimiento de soledad, favoreciendo esto el consumo de ansiolíticos 
y tranquilizantes. Las quejas y propuestas de mejora de las PC del ámbito rural a las medidas de apoyo en el hospital, se orientaron fundamentalmente a la información, la gestión de citas-circuitos-traslados, al derecho a proteger la intimidad y a los apoyos sociales.

Se identifica así, la necesidad de orientar las medidas de apoyo a las PC en el hospital en función del ámbito del que provengan, ya que como se ha comprobado las necesidades de cada una son distintas en función de las alteraciones personales provocadas por el cuidado, estando estas influenciadas en gran medida por los apoyos recibidos en su ámbito habitual y por las dificultades encontradas para el cuidado. Este resultado está en consonancia con el estudio realizado por Lorenzo y $\mathrm{col}^{23}$ sobre las necesidades percibidas por los cuidadores informales en función del hábitat de residencia rural o urbano, donde concluye que éste es un factor muy influyente y desencadenante de desequilibrios, de forma que las personas que viven en zonas rurales presentan menores posibilidades reales de acceso a los recursos, así como mayor desconocimiento de los mismos.

También se ha identificado la necesidad de prestar más apoyos, tanto sanitarios como sociales en el medio rural, lo que corrobora la propuesta realizada por Durán en el capítulo "Entorno, hábitat: medio rural y medio urbano" del Libro blanco sobre envejecimiento activo ${ }^{24}$ en la que defiende que sería necesario el desarrollo de estrategias para cubrir el desequilibrio en servicios sociales básicos y de bienestar existente en relación con el medio urbano.

Por otra parte también hay que considerar las dificultades encontradas por las PC del medio rural a consecuencia de la dispersión y la distancia que a veces puede existir entre el domicilio y el centro de atención sanitaria. Como se recoge en la bibliografía, ésta es una desprotección que se vive y se padece ${ }^{25}$.

Uno de los elementos mejor valorados por las PC, sobre todo por las del ámbito rural, fue la personalización del cuidado a través de la asignación de enfermera y auxiliar referentes. Esta asignación favo- reció las relaciones interpersonales y la humanización del cuidado, ambos aspectos destacados por Arredondo-González y Siles-González ${ }^{26}$ en su teoría de las relaciones interpersonales. En este sentido se puede considerar que la personalización del cuidado tiene un efecto terapéutico beneficioso, por el que se disminuye la carga psíquica de las PC incluidas en el Plan Funcional, y se aumenta la seguridad y el bienestar tanto de ellas como de las personas cuidadas, especialmente en el ámbito rural. Estas intervenciones son necesarias, como recogen Losada y $\mathrm{col}^{27}$ y López y $\mathrm{Crespo}^{28}$, para disminuir la morbilidad psicológica y los pensamientos disfuncionales generados por el cuidado prolongado, o García-González ${ }^{29}$ a través del programa "Rompiendo Distancias" en relación a la atención a personas dependientes del ámbito rural.

La implementación del Plan Funcional con medidas de apoyo a las PC en los dos hospitales granadinos, establece un nuevo marco de relación entre los profesionales del hospital y las PC dignifica a las PC realizando intervenciones multidisciplinares para apoyarlas individualmente, tanto de forma preventiva como de soporte, con los consiguientes beneficios tanto para ellas como para las personas dependientes. Esta iniciativa está en la línea expresada en los principios generales a favor de las personas dependientes emitidos por el Comité de Ministros Europeos a los estados miembros, en el que se recomienda a los gobiernos de los estados diseñar estrategias de apoyo a las PC basadas en el conocimiento y comprensión de sus problemas y necesidades $^{30}$.

Como conclusión, se puede afirmar que el ámbito donde se desenvuelve habitualmente la vida de las PC hace que sus percepciones del cuidado y sus necesidades en el hospital presenten algunas diferencias, lo que hace necesario que las medidas de apoyo en el hospital se adapten en función del ámbito al que pertenecen. Asimismo se han de redefinir las prioridades de las medidas y sería necesario centrarse en solucionar el problema del descanso nocturno e incluir medidas adicionales en 
función de la situación familiar, especialmente en aquellas que presentan niveles importantes de disfunción familiar. Para ello se erige como elemento fundamental la personalización de cuidados, debiendo realizar en los casos precisos una valoración focalizada de la PC por la enfermera referente con todas las actuaciones que se deriven de ella, una vez identificados los problemas existentes.

\section{Agradecimientos}

A todas las personas cuidadoras que tan amablemente nos contaron su realidad y a los profesionales colaboradores de los hospitales Virgen de las Nieves y Baza.

\section{BIBLIOGRAFÍA}

1. Bazo Royo, MT. El desafío del envejecimiento en los sistemas sanitarios y sociales de España y Europa. $1^{\text {a }}$ edición. Editorial: Nau Llibres (Edicions Culturals Valencianes, S.A.) 2012.

2. Encuesta sobre Discapacidades, Autonomía personal y situaciones de Dependencia (EDAD 2008). Instituto nacional de estadística. Disponible en http://www.ine.es/ jaxi $/$ menu.do? $\mathrm{L}=0 \&$ type $=$ pcaxis $\&$ path $=/ \mathrm{t} 15 /$ p418\&file=inebase. Consultada 02-12-2012.

3. Abellán García A, Pérez díaz J, Esparza Catalán C, Castejón Villarejo P, Ahmed-Mohamed K. Discapacidad y dependencia en Andalucía con base en la Encuesta sobre Discapacidad, Autonomía personal y situaciones de Dependencia (EDAD) 2008. Instituto de Estadística y Cartografía de Andalucía. Sevilla 2012: 22-28.

4. HernándeZ G, MELÉndez MC. Envejecimiento poblacional, dependencia y previsión de la autoprotección. Revista de investigaciones Políticas y Sociológicas (RIPS) 2010; 19: 137160 .

5. Decreto 137/2002 de 30 de Abril de apoyo a las familias andaluzas. BOJA n ${ }^{\circ} 52$ de 4 de mayo de 2002 (57) 7:127-134.

6. Ley 39/2006 de 14 de diciembre de promoción de la autonomía personal y atención a las personas en situación de dependencia y a las familias.BOE $\mathrm{n}^{\circ} 299$ de 15 de diciembre de 2006.

7. Andrade G, Pérez I. Apoyo de enfermería a las actividades que realizan los cuidadores informales en el ámbito hospitalario. Desarrollo Cientif Enferm 2007; 15: 52-55.
8. Quero Rufí́n A. Los Cuidadores familiares en el Hospital Ruiz de Alda de Granada. Tesis Doctoral. Universidad de Granada 2007.

9. Esparza Catalán, C. Discapacidad y dependencia en Andalucía. Madrid, Informes Portal Mayores, $n^{\mathrm{o}} 110$. Fecha de publicación: 17/08/2011. Disponible en: http://www. imsersomayores.csic.es/documentos/documentos/pm-discapacidadandalucia-01.pdf. Consultado el 14-09-2012.

10. Durán Heras MA. Las personas mayores en el medio rural y urbano. Ministerio de Sanidad y Política Social, Secretaría General de Política Social y Consumo. Instituto de Mayores y Servicios Sociales (IMSERSO) 2011: 461-465.

11. Fernández de Larrinoa Palacios P, Martínez Rodríguez S, Ortiz Marqués N, Carrasco Zabaleta M, Solabarrieta Eizaguirre J, Gómez Marroquín I. Auto percepción del estado de salud en familiares cuidadores y su relación con el nivel de sobrecarga. Psicothema 2011; 23: 388-393.

12. Vázquez-Sánchez MA, Aguilar-Trujillo MP, Estébanez-Carvajal FM, Casals-VázQuez C, CasalsSÁnchez JL, Heras-PÉrez MC. Influencia de los pensamientos disfuncionales en la sobrecarga de los cuidadores de personas dependientes. Enferm Clin 2012; 22: 11-17.

13. Masanet Ripoll, E. La Parra Casado, D. Los impactos de los cuidados de salud en los ámbitos de vida de las personas cuidadoras. RES. Revista Española de Sociología 2009; 11: 1331.

14. Minguela Recover MA, Camacho Ballesta JA. Dependencia: Cuidados formales e informales en Andalucía. Diferencias entre el ámbito rural y urbano. III Congreso anual de la REPS (Red Española de Política Social). Universidad Pública de Navarra. Pamplona 24-26 de noviembre de 2011.

15. Camarero L, Cruz F, González M, Del Pino JA, Oliva J, SAmpedro R. La población rural de España: de los desequilibrios a la sostenibilidad social. Extracto población rural en España. Fundación La Caixa. Barcelona 2009: 29-41 y 79-98.

16. Gorlat Sánchez B, Martín Berrido, M, Alcántara Díaz C, Calle García MJ, Cueto Rejón E, GonzÁlez Guerrero L et al. Plan funcional de personas cuidadoras en el hospital Virgen de las Nieves de Granada. Documento electrónico. 2010. [consultado 03-072012]: Disponible en: http://www.google. es/url?sa=t\&rct=j\&q=plan\%20funcional $\% 20$ de $\% 20$ personas $\% 20$ cuidadoras $\% 20$ en $\% 20$ el\%20hospital\%20\&source=web\&cd=1\&ved= 0CC4QFjAA\&url=http $\% 3 \mathrm{~A} \% 2 \mathrm{~F} \% 2 \mathrm{Fwww} . \mathrm{hvn}$. es $\% 2$ Fenfermeria $\% 2$ Fficheros $\% 2$ Fplan_fun- 
cional_definitivo_con_isbn_del_pch.pdf\&ei= K4jAUOiOMoqN0QHH54HYDw\&usg=AFQjCN HtxI8hryoccIMmilhxkGZG6E_Nww

17. Gorlat-Sánchez B, Sola-Peral A, Peinado-Gorlat P, Pérez-Sáez F, López-Hidalgo J, Alarcón-Cardona J et al. Medidas de apoyo a las personas cuidadoras en el hospital de Baza. Granada. 2012.

18. Strauss A, Corbin J. Bases de la investigación cualitativa. Técnicas y procedimientos para desarrollar la Teoría Fundamentada. Colombia: Editorial Universidad de Antioquía 2002: 13-28.

19. Bellón JA, Delgado A, Luna JD, Lardelli P. Validez y fiabilidad del cuestionario de función familiar Apgar-familiar. Aten Primaria 1996; 18: 17-31// 289-295.

20. Gálvez J, Ras E, Hospital I, Vila A. Perfil del cuidador principal y valoración del nivel de ansiedad y depresión. Aten Primaria 2003; 31:144-145.

21. De la Revilla L, Bailón E, De Dios luna J, Delgado A, Prados MA, Freitas L. Validación de una escala de apoyo social funcional para su uso en la consulta del médico de familia. Aten Primaria 1991; 8: 688-692.

22. Corbin JA, Strauss A. Basics of qualitative research, 3rd ed. Thousand Oaks, CA: Sage. 2008.

23. Lorenzo Otero T, Maseda Rodríguez A, Millán CALEnTI JC. La dependencia en las personas mayores: Necesidades percibidas y modelo de intervención de acuerdo al género y al hábitat. Instituto Gallego de Iniciativas Sociales y Sanitarias 2008.

24. DuRÁN MA. IMSERSO. Libro blanco sobre envejecimiento activo. Entorno, hábitat: medio rural y medio urbano. Capítulo 13. Pag 523.
Disponible en:http://www.imserso.es/imserso_01/envejecimiento_activo/libro_blanco/index.htm. Consultado 10-2-2013.

25. La Promoción de la Salud en el Medio Rural: Necesidades y Demandas Expresadas por las Mujeres. Documentos técnicos de salud pública, $\mathrm{n}^{\circ}$ 88. Instituto de Salud Pública. Consejería de Sanidad. Comunidad de Madrid. Diciembre de 2002.

26. Arredondo-GonzÁlez CP, Siles-GonzÁlez J. Tecnología y Humanización de los Cuidados: Una mirada desde la Teoría de las Relaciones Interpersonales. Index Enferm 2009; 18: 3236. Disponible en: http://www.index-f.com/ index-enfermeria/v18n1/r6624.php. Consultado 2-12-2012.

27. Losada Baltar A, Montorio Cerrato I, Izal Fernández de Trocóniz M, Márquez González M. Estudio e intervención sobre el malestar psicológico de los cuidadores de personas con demencia. El papel de los pensamientos disfuncionales. Premio IMSERSO "Infanta Cristina”. Ministerio de Trabajo y Asuntos Sociales. Secretaría de Estado de Servicios Sociales, Familias y Discapacidad. 2005.

28. LóPEZ J, CRESPO M. Intervenciones con cuidadores de familiares mayores dependientes: una revisión. Psicothema 2007; 19: 72-80.

29. GARcíA-GonZÁlez J. Rompiendo distancias: un programa integral para prevenir y atender la dependencia de las personas mayores en el medio rural. Rev Esp Geriatr Gerontol 2005; 40: 22-33.

30. Consejo de Europa. Recomendación $n^{\circ}$ (98) 9 del Comité de Ministros a los Estados Miembros, relativa a la dependencia. Anexo a la Recomendación n⿳ำ $\mathrm{R}$ (98) 9. Estrasburgo: Consejo de Europa. 1998. 